\title{
Comparative Studies of Graphitic Material Interaction with Flame Retardant 4 (FR-4) Substrate for Miniature Electrochemical-based $\mathrm{pH}$ Sensor
}

\author{
Khi Khim Beh, ${ }^{1}$ Asrulnizam Abd Manaf, ${ }^{1 *}$ Chuan Wei Oo, ${ }^{2}$ \\ Khi Poay Beh, ${ }^{3 * *}$ Fong Kwong Yam, ${ }^{3}$ and Kazuaki Sawada ${ }^{4}$ \\ ${ }^{1}$ Collaborative Microelectronic Design Excellence Center (CEDEC), Universiti Sains Malaysia, \\ SAINS@USM, 11900 Bayan Lepas, Penang, Malaysia \\ ${ }^{2}$ School of Chemical Sciences, Universiti Sains Malaysia, 11800 Minden, Penang, Malaysia \\ ${ }^{3}$ School of Physics, Universiti Sains Malaysia, 11800 Minden, Penang, Malaysia \\ ${ }^{4}$ Department of Electrical and Electronic Information Engineering, Toyohashi University of Technology, \\ 1-1 Hibarigaoka, Tempaku-cho, Toyohashi, Aichi 441-8580, Japan
}

(Received November 22, 2019; accepted April 30, 2020)

Keywords: $\mathrm{pH}$, electrochemical sensor, graphene oxide, reduced graphene oxide, PCB

In this paper, we present studies of graphitic materials clad on a working electrode (WE) for a miniaturised three-electrode electrochemical-based $\mathrm{pH}$ sensor. The sensor was fabricated on a flame retardant 4 (FR-4)-based printed circuit board (PCB), with additional layers of electroplated nickel $(\mathrm{Ni})$ and gold $(\mathrm{Au})$ to shield the base copper cladding from corrosion. In this study, Au, graphene oxide (GO)/polyvinyl alcohol (PVA), and reduced graphene oxide $(\mathrm{rGO}) / \mathrm{PVA}$ were used for fabricating WEs, then each electrode was analysed for its capability to produce a reversible redox reaction. Cyclic voltammetry (CV) measurements showed that all the fabricated WEs can produce a reversible redox reaction, with different peak currents. This showed that the functional groups within GO plays an important role in the $\mathrm{pH}$ sensing mechanism with their open-circuit potential varying with different pHs. The optimised fabricated WEs were integrated with reference and counter electrodes, then characterised for $\mathrm{pH}$ sensor application. Among all the sensors, GO/PVA showed the highest sensing capability of $8.3 \mathrm{mV} / \mathrm{pH}$.

\section{Introduction}

In recent years, electrochemical (EC)-based sensing technology has offered analytical diagnosis measurement systems of lesser complexity, smaller form factor, and better portability. ${ }^{(1)}$ These make the technology a better alternative to conventional bulky setups and suitable for quick and on-site testing. ${ }^{(2)}$ With the rapid growth and demand for in situ and portable EC sensors, further miniaturisation of the electrodes into microelectrodes permits a significant density of sensors to be packed in a small volume, ${ }^{(3)}$ which would be advantageous for realizing multiprobing capability. ${ }^{(4)}$ Despite the successful miniaturisation of the electrodes, not all

\footnotetext{
*Corresponding author: e-mail: eeasrulnizam@usm.my

** Corresponding author: e-mail: behkhipoay@usm.my

https://doi.org/10.18494/SAM.2020.2703
} 
sensors were created equal, as electrode modification was necessary to suit the environment of the analyte to be measured. As an example, modified, working electrodes (WEs) should be compatible with biological specimens or should cater to environmental sensing, such as pollution sample measurement. ${ }^{(5)}$ With proper modification, a portable EC sensor has advantageous for various fields such as medicine, biochemistry, analytical chemistry, and environmental monitoring. ${ }^{(6-9)}$

Currently, silicon, ceramic, and glass substrates are commonly used in the microfabrication of EC sensors. These substrates are hard but brittle, and thus require handling with utmost care. This apparent drawback limits their practical uses in the field owing to the additional care that needs to be done. ${ }^{(10,11)}$ Paper-based substrates have merits in terms of higher productivity. However, their rugged structure surface will require sensor electrode modifications, which becomes a limitation. ${ }^{(12)}$ On the other hand, the flame retardant 4 (FR-4) substrate has advantages in terms of mechanical strength and easy processability with simple facilities. Additionally, the copper $(\mathrm{Cu})$-cladded FR-4 board has been extensively used in printed circuit board (PCB) industries. The technology is readily available and its processing procedures have been well developed. These advantages would be very suitable for producing a simple structure of the EC sensor using FR-4 as a substrate. ${ }^{(13-15)}$ Besides, the matured PCB technology will enable the incorporation of numerous functional blocks along with the sensor. One example of such blocks is the measurement/readout circuitry that provides instant on-site results. With the advancement in surface-mount device (SMD) technologies, constructing the measurement/readout circuitry within a tight dimension became possible. With this development, additional functional blocks in the form of packaging can be fabricated as well. Functional packaging blocks such as microfluidic channels can be integrated into the active sensor so that an analyte can be quantitatively analysed. Advanced development of the PCB technology will lead to the next-generation PCB technology from a system on board (SoB) to a system on film (SoF) using printable electronics. ${ }^{(16)}$

Graphene oxide (GO) is a unique and essential material in the application of sensors. Its abundant oxygen-containing functional groups can be either chemically manipulated or used as an adduct in sensing an assortment of materials. ${ }^{(17,18)}$ GO has been used in detecting biomaterials including DNA, bacteria, and so on. Moreover, it has been used in detecting ions ranging from hydrogen ions $\left(\mathrm{H}^{+}\right)$to heavy metal ions. ${ }^{(19)}$ The simplest ion detection would be $\mathrm{H}^{+}$detection, which is also related to $\mathrm{pH}$. The $\mathrm{pH}$ of a solution is essential in various chemical reactions in the lab or nature. Monitoring the $\mathrm{pH}$ of an environment is essential in determining the sustainability of life. This would require maintenance-free and long-life-span portable EC sensors. In this work, an EC sensor with a modified WE was fabricate in-house and then characterised using several buffer solutions of various $\mathrm{pHs}$. The sensors were made from a $\mathrm{Cu}-$ cladded board. The choices of electrode material were GO/polyvinyl alcohol (PVA) and reduced graphene oxide (rGO)/PVA. An Au electrode was used as the control electrode to compare the modified electrode performances. In this study, we show the capability of the EC sensor in detecting $\mathrm{pH}$ with minimal electrode surface modification on an FR-4 substrate. 


\section{Materials and Methods}

\subsection{Chemicals and materials}

All chemicals used were of analytical reagent grade. Graphite flakes were purchased from Ding Sheng Xin Chemical Industry. Potassium permanganate $\left(\mathrm{KMnO}_{4}\right)$ and sulphuric acid $\left(\mathrm{H}_{2} \mathrm{SO}_{4}\right)$ were purchased from QReC. Phosphoric acid $\left(\mathrm{H}_{3} \mathrm{PO}_{4}\right)$ was acquired from (ChemPur), and potassium hexacyanoferrate trihydrate $\left(\mathrm{K}_{4} \mathrm{Fe}(\mathrm{CN})_{6}\right)$ was purchased from Sigma Aldrich. Stock solutions of various pHs were prepared by mixing acetic acid and sodium hydroxide $(\mathrm{NaOH})$, with a few drops of universal indicator solution from $\mathrm{R} \& \mathrm{M}$ to visualise the changes in $\mathrm{pH}$. Both acetic acid and $\mathrm{NaOH}$ were acquired from $\mathrm{R} \& \mathrm{M}$. The reduction of $\mathrm{GO}$ was carried out using ascorbic acid purchased from $\mathrm{HmbG}$. Gold (Au) and silver (Ag) plating solutions were purchased from Spa Plating UK.

\subsection{Instrumentations}

The Gamry Interface 1000 potentiostat connected to a computer was used to conduct cyclic voltammetry $(\mathrm{CV})$ and open circuit potential $(\mathrm{OCP})$ measurements. For the synthesis of GO and rGO, a hotplate stirrer (JoanLab, model HSC-19T) was used. GO and rGO were washed using the Ohaus FC5718R Multi Pro Frontier 5000 centrifuge system from Novatech. The surface morphology and the elemental composition of the electrode materials were determined by field emission scanning electron microscopy (FESEM, FEI Nova NanoSEM 450) with energy-dispersive X-ray spectroscopy (EDX, Oxford). The vibrational modes of GO and rGO were characterised using a Renishaw InVia Raman spectrometer at an incident laser power and wavelength of $20 \mathrm{~mW}$ and $633 \mathrm{~nm}$, respectively.

\subsection{Fabrication of $\mathrm{pH}$ sensor platform}

Figure 1 shows the overall fabrication process of the $\mathrm{pH}$ sensor. The $\mathrm{pH}$ sensor pattern was designed using DipTrace software and printed on an inkjet film as the master mask. A negative photoresist film was laminated on a clean FR-4 board at a temperature of $110{ }^{\circ} \mathrm{C}$. Then, the master mask was placed on the photoresist surface. Next, an ultraviolet (UV) light from a UV exposure machine was shone on the master mask for $20 \mathrm{~s}$. This hardened the photoresist as a result of polymeric cross-linking. The hardened photoresist protected the $\mathrm{Cu}$ underneath from subsequent chemical etching. After that, the master mask was removed, and the board was immersed into an aqueous developer that contained $\mathrm{HCl}$. The aqueous developer washed off the unexposed region, subsequently revealing the electrode pattern. Since only the photoresist was developed, while the entire $\mathrm{Cu}$ surface was untouched, the FR-4 board was rinsed with deionised (DI) water and then immersed in $\mathrm{FeCl}_{3}$. The $\mathrm{FeCl}_{3}$ etches the exposed $\mathrm{Cu}$, which leads to the formation of $\mathrm{Cu}$ traces that resembled the electrode patterns. The excess $\mathrm{FeCl}_{3}$ on the FR-4 board was rinsed thoroughly with DI water again, followed by immersion into a stripper solution to remove the remaining photoresist on the copper surface. 


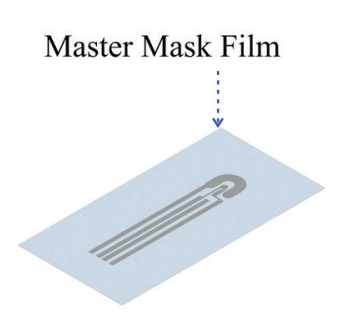

(a)

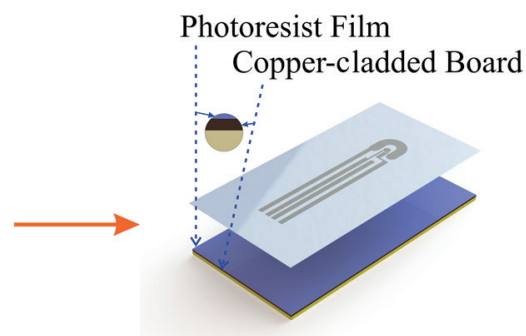

(b)

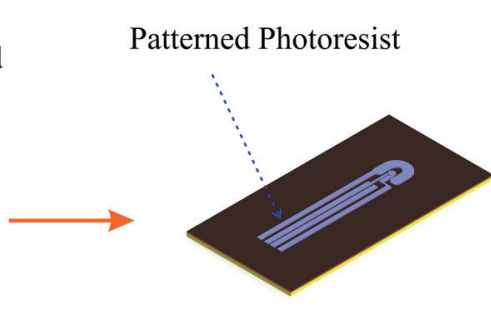

(c)

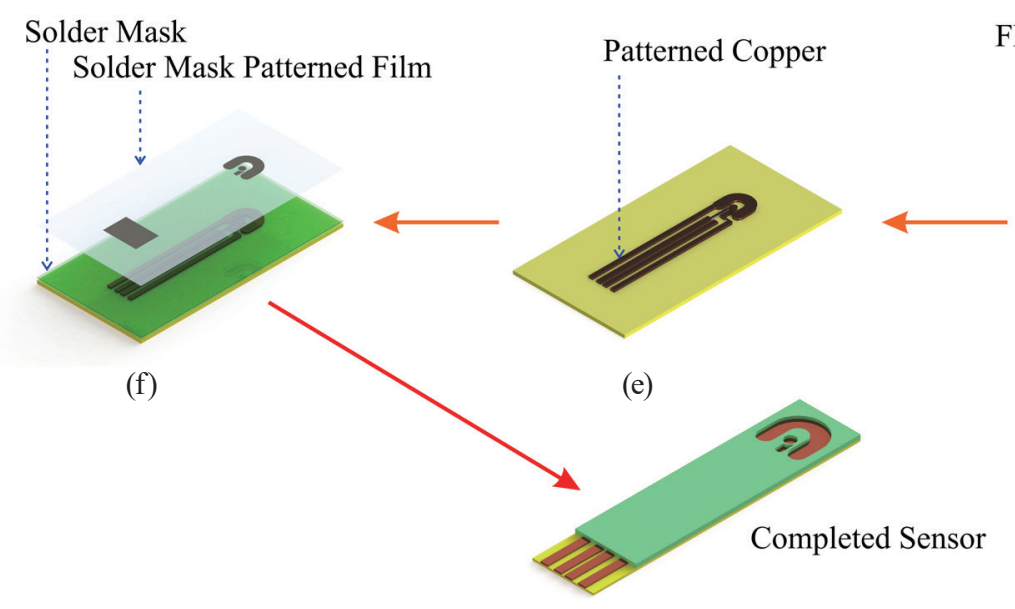

FR-4 Substrate Photoresist patterned

(g)

Fig. 1. (Color online) Overall fabrication of $\mathrm{pH}$ sensor.

\subsection{Configuration and operation of $\mathrm{pH}$ sensor}

The in-house-fabricated $\mathrm{pH}$ sensor adopts the conventional three-electrode topology, i.e., working, counter, and reference electrodes (WE, CE, and RE, respectively) as shown in Figs. 2(a) and 2(b). A schematic representation of the $\mathrm{pH}$ sensing setup is shown in Fig. 2(c). The pH sensor was connected to a USB female Type-A adapter, which is commonly available. Our $\mathrm{pH}$ sensor operates similarly to any other three-electrode-based $\mathrm{pH}$ sensor, with the exception that the former has been miniaturised. The sensing mechanism of the $\mathrm{pH}$ sensor is illustrated in Fig. 2(d). Notably, the surface area of CE is larger than that of WE in order to prevent an overpotential from happening during operation. Besides, CE prevents current flowing into RE. RE provides a stable potential in the circuit. Thus, it allows potential measurements at WE and RE to happen. Finally, WE is the core sensing component throughout the sensor. WE measures the redox reaction with an electrolyte. When potential is applied from a lower to a higher value within the potential range, the current peak value is obtained when oxidation happens and vice versa. 


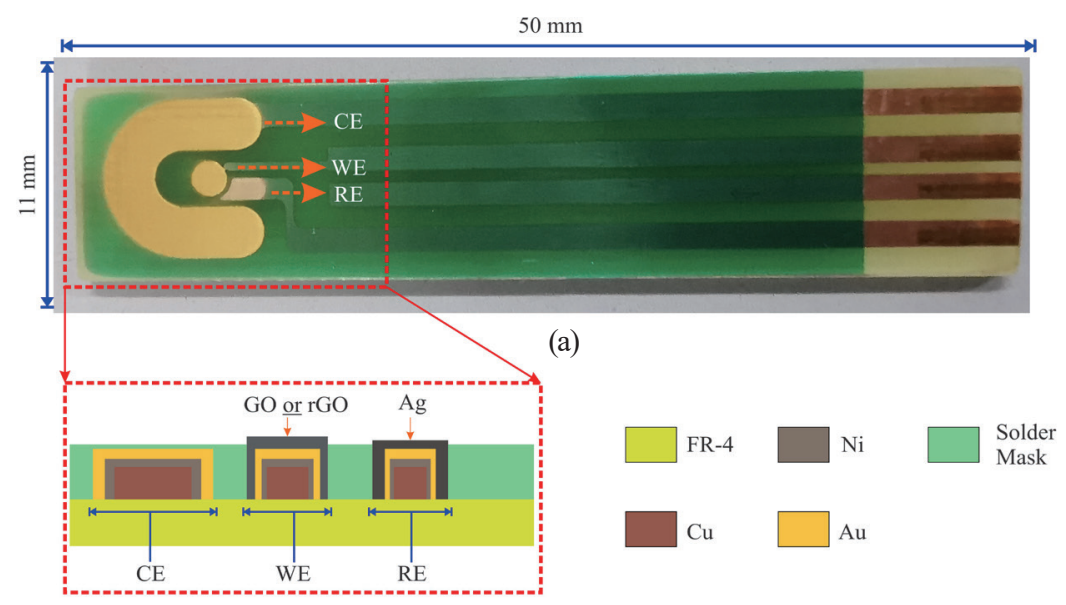

(b)

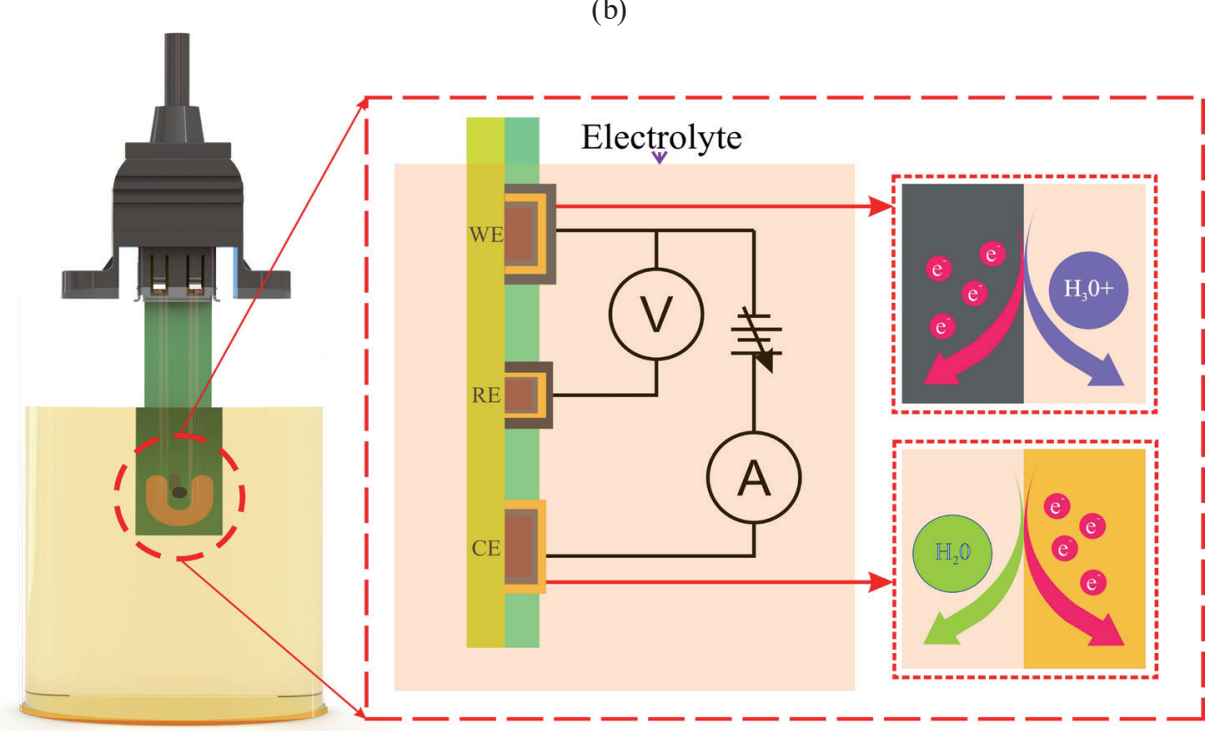

(c)

(d)

Fig. 2. (Color online) (a) Digital photograph of base platform of $\mathrm{pH}$ sensor, (b) cross-sectional view of electrodes, (c) experimental setup for $\mathrm{pH}$ sensing, and (d) sensing mechanism of $\mathrm{pH}$ sensor.

\subsection{Metal electrode electroplating process}

The as-fabricated $\mathrm{pH}$ sensor platform consisted of only $\mathrm{Cu}$ traces, which is prone to oxidation and requires protective layers. In this work, Au served such purposes. However, owing to the poor adhesion of $\mathrm{Au}$ on $\mathrm{Cu}$, this necessitated an intermediate buffer layer. Nickel (Ni) was chosen for such a purpose. Au and Ni were deposited on the as-fabricated sensor platform using an electroplating processer. The sequence of metals to be electroplated was Ni followed by Au. For RE, an extra layer of Ag was electroplated. For the entire electroplating process, the anode was made of stainless steel, while the counter electrode was the sensor platform consisting of $\mathrm{Cu}$ traces. Aside from that, the sensor was thoroughly cleaned using DI water and isopropyl 
alcohol (IPA) before beginning the next electroplating step. The electroplating process was galvanostatic as opposed to potentiostatic, with each metal having their respective electroplating current. The electroplating parameters of each metal layer are summarised in Table 1.

\subsection{GO and rGO synthesis}

In this work, GO was synthesised by Tour's method. In brief, $500 \mathrm{mg}$ of pure graphite flakes were mixed with $\mathrm{KMnO}_{4}$ at a weight ratio of 1:6. Next, a mixture of $\mathrm{H}_{2} \mathrm{SO}_{4}$ and $\mathrm{H}_{3} \mathrm{PO}_{4}$ at a weight ratio of 4:1 was prepared in a separate beaker. The mixture of acids was stirred for 15 mins. Then, a solid mixture of graphite flakes and $\mathrm{KMnO}_{4}$ was added to the acid mixture steadily under continuous stirring while maintaining the mixture's temperature at $45^{\circ} \mathrm{C}$. After adding the solid mixture into the acid, the reaction mixture was continuously stirred for $72 \mathrm{~h}$. This allowed sufficient time for the oxidation to occur. The colour of the mixture gradually transitioned from purplish green to light brown after $72 \mathrm{~h}$, indicating that graphite flakes have been oxidised. Since the mixture contained concentrated acids, it was diluted prior to other processes by pouring the acidic mixture into ice cubes, which melted owing to the subsequent exothermic reaction, thereby increasing the solution temperature back to room temperature. The absence of additional heating prevented the structural damage of graphite oxide flakes. The dilution of the acidic mixture resulted in a total volume of $500 \mathrm{ml}$. Next, to terminate the oxidation of graphite, $10 \mathrm{ml}$ of $30 \% \mathrm{H}_{2} \mathrm{O}_{2}$ was added into the mixture, which turned the colour of the solution into golden yellow, indicating the successful oxidation of graphite flakes. At this stage, graphite oxide was obtained. However, to convert them into GO, the former was centrifuged multiple times at $12000 \mathrm{rpm}$ for $4 \mathrm{~h}$. The centrifugal force during centrifugation exfoliated the graphite oxide into GO sheets. Additionally, the residue contaminants from the oxidation reactions were removed as well. The washing of GO ended when the solution reached $\mathrm{pH}$ 6. Next, the GO slurry was poured into a Teflon beaker and freeze-dried for $48 \mathrm{~h}$.

The rGO synthesis in this work involved the chemical reduction of GO. About $200 \mathrm{mg}$ of dried GO was dispersed in $100 \mathrm{ml}$ of DI water through an intense sonication process. After achieving uniform dispersion, the mixture was continuously stirred at a speed of $700 \mathrm{rpm}$ at ambient temperature. Next, $3 \mathrm{M} \mathrm{NaOH}$ was added into the GO solution until the mixture reached $\mathrm{pH}$ 9. Then, $1 \mathrm{~g}$ of ascorbic acid $\left(\mathrm{C}_{6} \mathrm{H}_{8} \mathrm{O}_{6}\right)$ was added into the mixture to chemically reduce GO. To hasten the reduction process, the mixture was heated to $95^{\circ} \mathrm{C}$ while continuously stirring for $2 \mathrm{~h}$. During this time, the brownish GO gradually turned black and precipitated. The black precipitate was filtered and washed several times with DI water.

Table 1

Sequence of electroplating and related parameters.

\begin{tabular}{|c|c|c|c|c|c|}
\hline $\begin{array}{l}\text { Electroplating } \\
\text { sequence }\end{array}$ & Metal & Target electrode & Electroplating bath & $\begin{array}{l}\text { Electroplating } \\
\text { current }(\mu \mathrm{A})\end{array}$ & $\begin{array}{l}\text { Electroplating } \\
\text { duration (min) }\end{array}$ \\
\hline 1 & $\mathrm{Ni}$ & WE, CE, RE & $\begin{array}{c}1 \mathrm{M} \text { nickel (II) sulphate }\left(\mathrm{NiSO}_{4}\right) \\
\text { and } 0.1 \mathrm{M} \text { of } \mathrm{HCl}\end{array}$ & 1000 & 15 \\
\hline 2 & $\mathrm{Au}$ & WE, CE, RE & Commercial gold electroplating solution & 350 & 15 \\
\hline 3 & $\mathrm{Ag}$ & RE only & Commercial silver electroplating solution & 7 & 5 \\
\hline
\end{tabular}




\subsection{Synthesis of GO/PVA and rGO/PVA composite and electrode modification}

The composite dispersion was prepared by mixing either GO or rGO dispersion in dissolved PVA. GO and rGO about $250 \mathrm{mg}$ each were separately added to two beakers containing $50 \mathrm{ml}$ of DI water. The mixture was homogenised through intense sonication. Afterwards, $1 \mathrm{~g}$ of PVA powder was added to the dispersion. To facilitate the dissolution of PVA, the solutions were heated to $90^{\circ} \mathrm{C}$ with vigorous stirring.

For the modification of the Au electrode, about $10 \mu \mathrm{L}$ of either GO/PVA or rGO/PVA was drop-casted on WE. The casting solutions were allowed to dry before $\mathrm{pH}$ sensing.

\section{Results and Discussion}

\subsection{Characterisation of modified electrode}

Figure 3 shows the FESEM images and EDX profile of the Au and Ag electrodes. There were several interesting morphological aspects to be highlighted. Firstly, the Au layers in

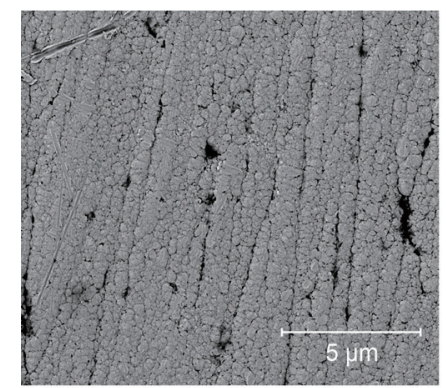

(a)

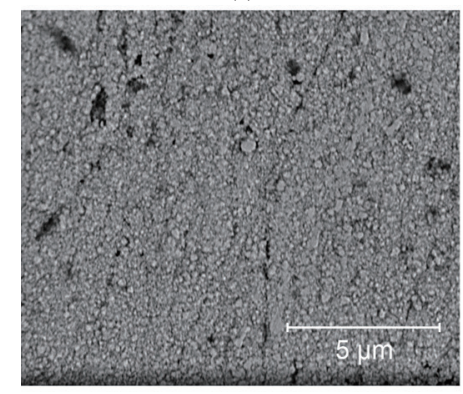

(c)

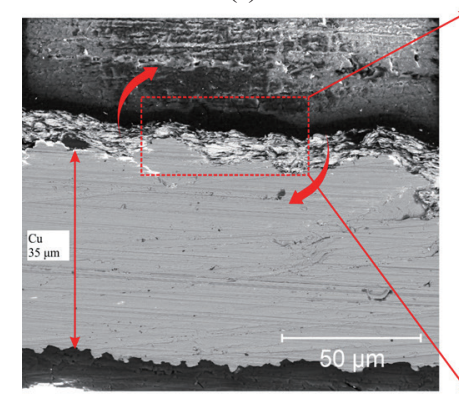

(e)

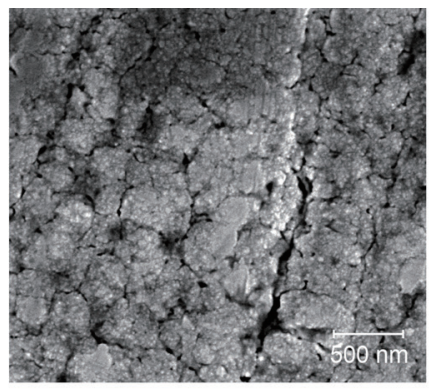

(b)

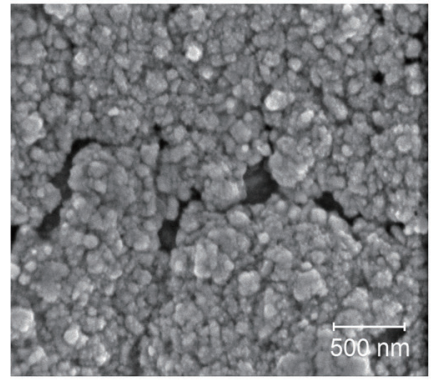

(d)

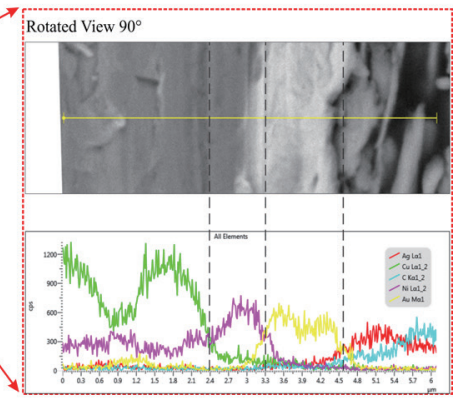

(f)

Fig. 3. (Color online) FESEM images of (a) and (b) Au surface, (c) and (d) Ag surface, (e) cross-sectional view of $\mathrm{pH}$ sensor electrode, and (f) estimated thickness of electroplated metal layers. 
Figs. 3(a) and 3(b) show surfaces uniformity decorated with nanostructures that resembled an ensemble of $\mathrm{Au}$ nanoparticles. The formation of such surfaces can be attributed to the microampere range of galvanostatic electrodeposition and the presence of a Ni layer. The latter served as an adhesion promoter for the former while being structurally compatible with that of $\mathrm{Cu}$ traces. The nanostructured surface of the Au layer would be advantageous for subsequent electrode modification owing to its higher surface-to-volume ratio that enables it to have a more significant chemical interaction, in addition to being a corrosion-resistant material. Next, Figs. 3(c) and 3(d) illustrate the electroplated Ag layer on a Au surface. Note that Ag only serves as RE and does not participate in any chemical reaction that occurs in WE and CE. Although the surface feature of $\mathrm{Ag}$ was similar to that of $\mathrm{Au}$, one notable difference was the distinct separation of Ag grains, which appeared to be less packed than Au. As the Ag grains possessed excellent adhesion to the Au layer, this allows RE to perform well in this miniaturised $\mathrm{pH}$ sensor. Figure 3(e) shows a cross section of the $\mathrm{pH}$ sensor platform, particularly at RE. The presence of several layered materials can be confirmed from their contrast differences. A more detailed morphology can be seen when a portion of Fig. 3(e) was magnified and displayed in Fig. 3(f). With the aid of the EDX line scan superimposed onto the figure, the thickness of each layer can be estimated. The images were further corroborated by the EDX mapping measurement. The estimated thicknesses were as follows: $2 \mu \mathrm{m}$ for $\mathrm{Ni}$ and $0.5 \mu \mathrm{m}$ for $\mathrm{Au}$. Four micrometers of Ag was obtained despite the electroplating current being the smallest $(\sim 7$ $\mu \mathrm{A})$. The thick Ag layer was necessary to provide a stable potential reference during the sensor operation.

Figures 4(a) and 4(b) show the morphologies of GO/PVA and rGO/PVA, respectively. The absence of features resembling those of the Au layer suggests that the deposited GO/PVA and $\mathrm{rGO} / \mathrm{PVA}$ is highly compact. Between GO/PVA and $\mathrm{rGO} / \mathrm{PVA}$, the former shows a wrinkled texture that is analogous to that of wet tissue; meanwhile, the rGO/PVA sample possessed flakes with polygonal edges. As PVA is an electrical insulator and crystallises upon drying, they appeared as high-contrast granular structures decorating the surface of the sample. PVA served as a cross-linker as well as a binder in this study. The necessity of PVA can be rationalised by the inability of both casted GO and rGO to maintain its sheetlike structure upon immersion into

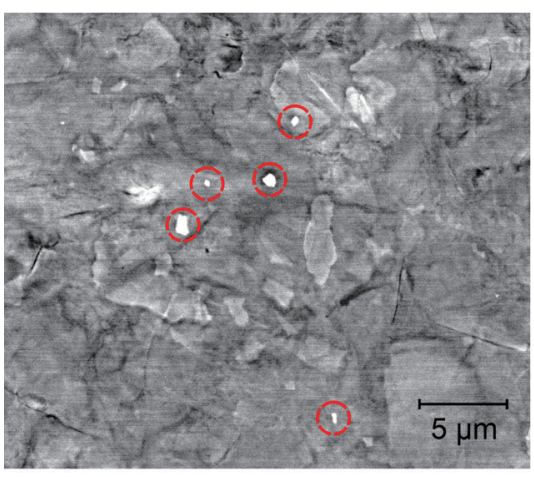

(a)

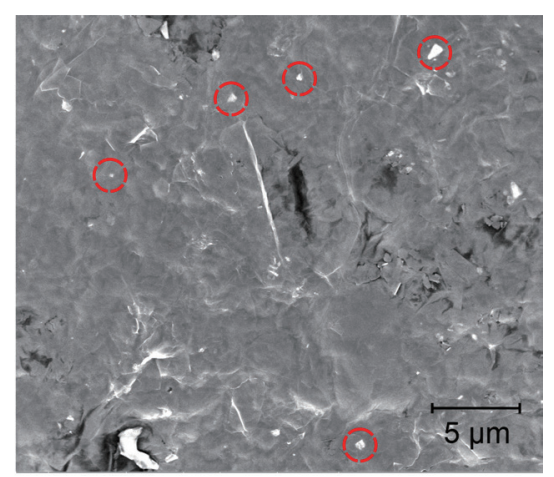

(b)

Fig. 4. (Color online) FESEM images of (a) GO/PVA and (b) rGO/PVA surfaces. 
the buffer solution. The PVA possessed numerous functional groups that could integrate with $\mathrm{GO}$ and $\mathrm{rGO}$, forming a continuous network that is less impervious to water.

\subsection{Raman spectroscopy studies}

Raman spectroscopy has been generally used to characterise carbon-based materials and nanostructures owing to its sensitivity towards the vibration of $\mathrm{C}-\mathrm{C}$ bonds. Figure 5 shows the Raman spectra of the GO/PVA and rGO/PVA. The two most prominent peaks for both graphitic materials were revealed to be in the range between 1100 and $1700 \mathrm{~cm}^{-1}$. The disorder bands (D bands) of GO/PVA and rGO/PVA were positioned at 1323 and $1343 \mathrm{~cm}^{-1}$, respectively. These peaks corresponded to the breathing mode of $k$-point phonons of $\mathrm{A}_{\mathrm{lg}}$ symmetry.

On the other hand, the in-plane vibration modes (G bands) of GO/PVA and rGO/PVA were discovered at $1594 \mathrm{~cm}^{-1}$, which is attributed to the $\mathrm{E}_{2 \mathrm{~g}}$ phonon of the $s p^{2}$ carbons. The ratio of D-to-G band peak intensities $I_{D} / I_{G}$ for GO/PVA was determined to be 1.1. Upon reduction, the $I_{D} / I_{G}$ for rGO/PVA was increased to 1.6 . The increase in the $\mathrm{D}$ band intensity can be attributed to the removal of functional groups on the basal plane of GO, which leads to the formation of point defects. Without a doubt, this significantly reduces the average crystallite size of the rGO.

\subsection{CV and scan rate dependence studies}

$\mathrm{CV}$ is extensively used to assess the performance of electrodes in facilitating redox reactions. Here, the electrode materials ( $\mathrm{Au}, \mathrm{GO} / \mathrm{PVA}$, and $\mathrm{rGO} / \mathrm{PVA})$ that made up the $\mathrm{pH}$ sensor were investigated. In general, secondary data derived from cyclic voltammograms provide some insights into the characteristics of the electrodes on the $\mathrm{pH}$ sensors. In this work, the scan rate of $\mathrm{CV}$ for all types of sensor ranged from 10 to $100 \mathrm{mV} / \mathrm{s}$. On the other hand, the chosen potential window for each sensor was unique, with $-0.4 \mathrm{~V}$ to $+0.6 \mathrm{~V}$ for the bare $\mathrm{Au}$ and GO/PVA pH sensors, and 0 to $0.4 \mathrm{~V}$ for the $\mathrm{rGO} / \mathrm{PVA} \mathrm{pH}$ sensor. As the test electrolyte,

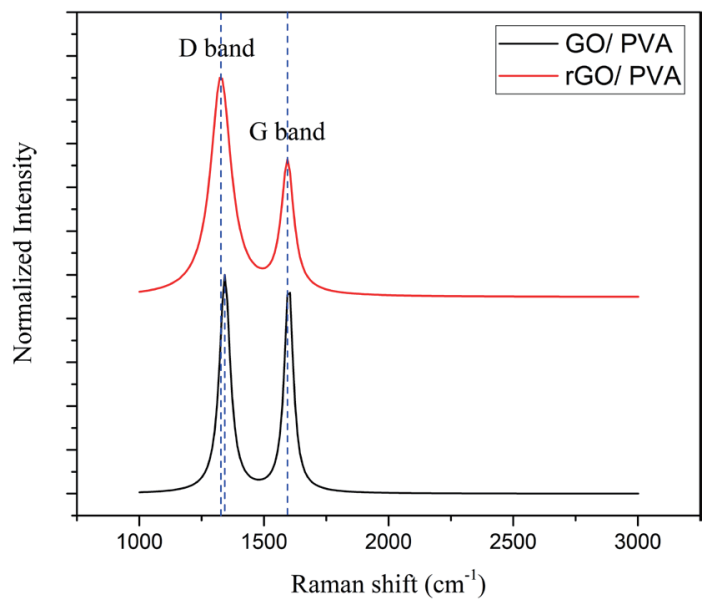

Fig. 5. (Color online) Raman spectra of GO/PVA and rGO/PVA. 
$0.05 \mathrm{M} \mathrm{K}_{4} \mathrm{Fe}(\mathrm{CN})_{6}$ was used for each sensor. In Figs. 6(a)-6(c), the voltammograms for all fabricated $\mathrm{pH}$ sensors are presented. Notably, all the voltammograms show reversible redox reactions. The potentials of the oxidation/reduction redox couple $\left(E_{p a} / E_{p c}\right)$ vary for each sensor. It was found that the $E_{p a} / E_{p c}$ values for the Au and GO/PVA sensors were almost similar, that is, $0.227 / 0.044$ and $0.223 / 0.042 \mathrm{~V}$, respectively. Interestingly, the $\mathrm{rGO} / \mathrm{PVA}$ sensor shows a significant deviation, where the $E_{p a} / E_{p c}$ was $0.186 / 0.085 \mathrm{~V}$. In terms of current magnitude, all sensors showed one common feature, that is, the current magnitude varies proportionally with scan rate. With regards to the peak redox current for each sensor, $\mathrm{Au}$ was found to have the highest, followed by GO/PVA, and finally, rGO/PVA. Here, anomalous differences between GO/PVA and rGO/PVA sensors exist, where the latter was perceived to be electrically more conductive than the former, and therefore, should exhibit a higher peak redox current. Such differences can be attributed to the sensing mechanism of the electrode material, where those

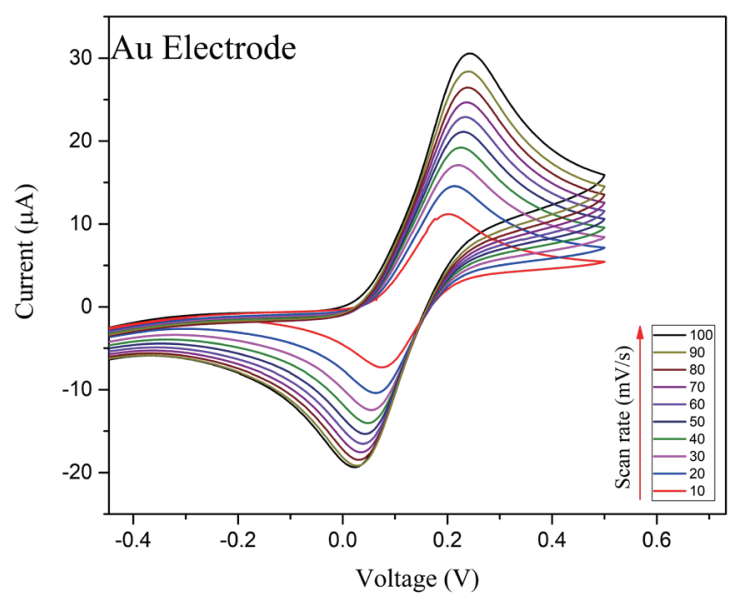

(a)

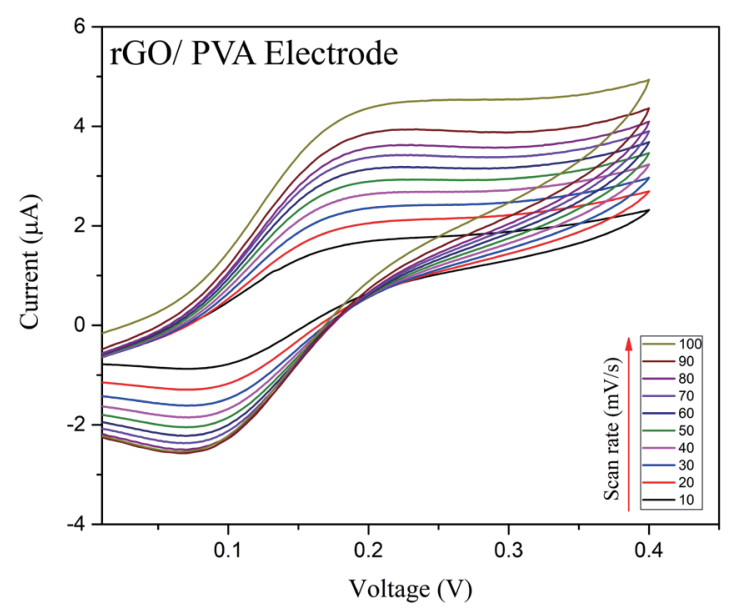

(c)

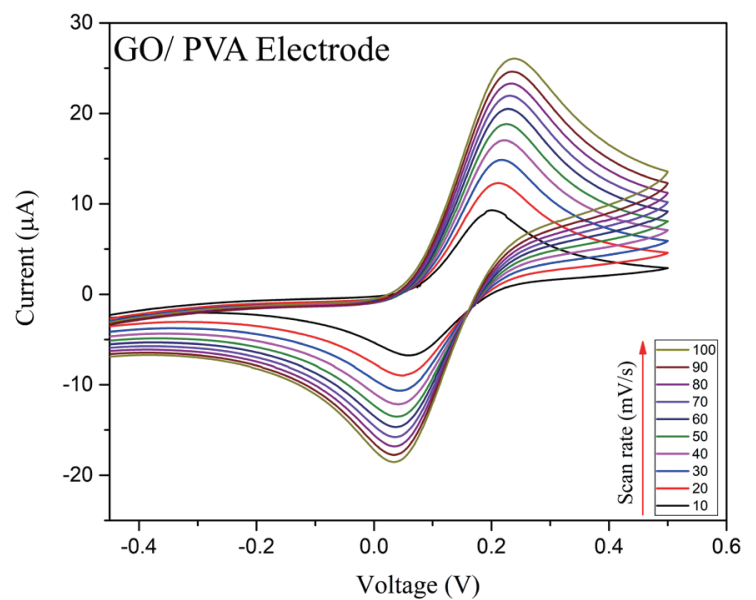

(b)

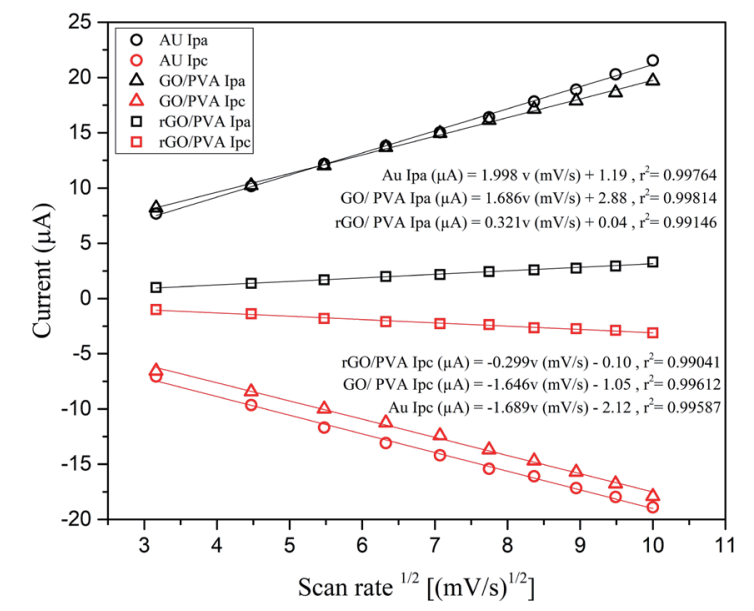

(d)

Fig. 6. (Color online) CV measurements of (a) Au, (b) GO/PVA, and (c) rGO/PVA on WE. (d) Linear regressions for $\mathrm{Au}, \mathrm{GO} / \mathrm{PVA}$, and $\mathrm{rGO} / \mathrm{PVA}$. 
with numerous functional groups dominate the redox reactions. Naturally, GO would possess assorted oxygen-containing functional groups within the basal plane as well as the edges, and thus better facilitates surface EC interactions. For rGO, the amount of functional groups was significantly reduced, while they were only present on the edges. This inadvertently hampered the surface EC interactions that led to a lower redox current. As for the Au electrode, owing to the absence of any chemical functional groups, the conductivity of Au played an important role, i.e., it determined the rate of charge transfer rate that resulted from surface chemical reactions.

Despite the unique behaviours for every sensor, their performances in facilitating singleelectron transfer from the $\mathrm{Fe}(\mathrm{CN})_{6}{ }^{4-/ 3-}$ system remained consistent. This is clearly shown in Fig. 6(d), where the regression coefficients $\left(r^{2}\right)$ for all sensors approach unity. The high linearity profile in Fig. 6(d) also suggests that the peak currents (both oxidation and reduction) followed the Randles-Sevcik model, where it can be mathematically expressed as

$$
I_{p}=\left(2.69 \times 10^{5}\right) \times(n)^{3 / 2} \times A D^{1 / 2} \times(C V)^{1 / 2},
$$

where $I_{p}$ is the peak current (in ampere), $n$ is the number of electrons, $A$ is the working electrode area $\left(\mathrm{cm}^{3}\right), D$ is the diffusion coefficient $\left(\mathrm{cm}^{3} \cdot \mathrm{s}^{-1}\right), C$ is the concentration $\left(\mathrm{mol} / \mathrm{cm}^{3}\right)$ of the solution, and $V$ is the scan rate $(\mathrm{V} / \mathrm{s})$. Another essential information that could be derived from the $\mathrm{CV}$ data would be the anodic/cathodic peak current ratio. This ratio illustrates the charge transfer kinetics under both anodic and cathodic conditions. Ideally, the anodic/cathodic peak current ratio under the given test environment in this work should be approximated to unity, as shown in Eq. (2), and the summary of values is shown in Table 2. The unity value illustrates the equilibrium charge transfer under anodic/cathodic conditions.

$$
I_{p a} / I_{p c} \approx 1
$$

\section{4 pH sensing studies}

Figure 7 shows the open circuit potential (OCP) results of the $\mathrm{Au}, \mathrm{GO} / \mathrm{PVA}$, and rGO/PVA electrodes using test reagents with $\mathrm{pHs} 4,7$, and 10. In general, $\mathrm{pH}$ is measured on the basics of the concentration (measured in $\mathrm{mol} / \mathrm{L}$ ) of hydrogen ions $\left(\mathrm{H}^{+}\right)$present within the test reagent. A high amount of $\mathrm{H}^{+}$ions would translate to low $\mathrm{pHs}$. The relationship between $\mathrm{H}^{+}$concentration and $\mathrm{pH}$ can be mathematically expressed as

$$
\mathrm{pH}=-\log _{10}\left[\mathrm{H}^{+}\right]
$$

Table 2

Peak current parameters for fabricated $\mathrm{pH}$ sensor material.

\begin{tabular}{lccc}
\hline Material & $I_{p a}(\mu \mathrm{A})$ & $I_{p c}(\mu \mathrm{A})$ & Ratio of $I_{p a} / I_{p c}$ \\
\hline $\mathrm{Au}$ & 15.4 & -14.1 & 1.1 \\
$\mathrm{GO} / \mathrm{PVA}$ & 14.9 & 12.7 & 1.2 \\
$\mathrm{rGO} / \mathrm{PVA}$ & 2.2 & 2.2 & 1.0 \\
\hline
\end{tabular}




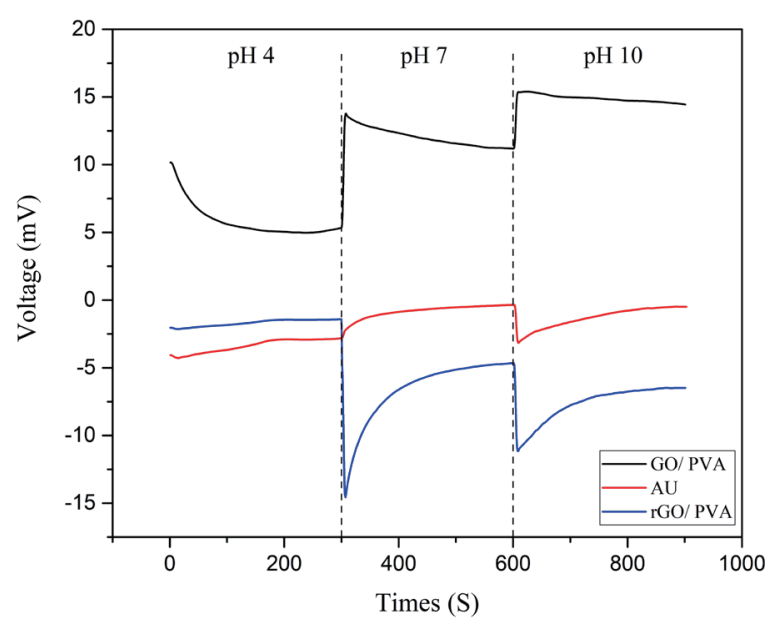

(a)

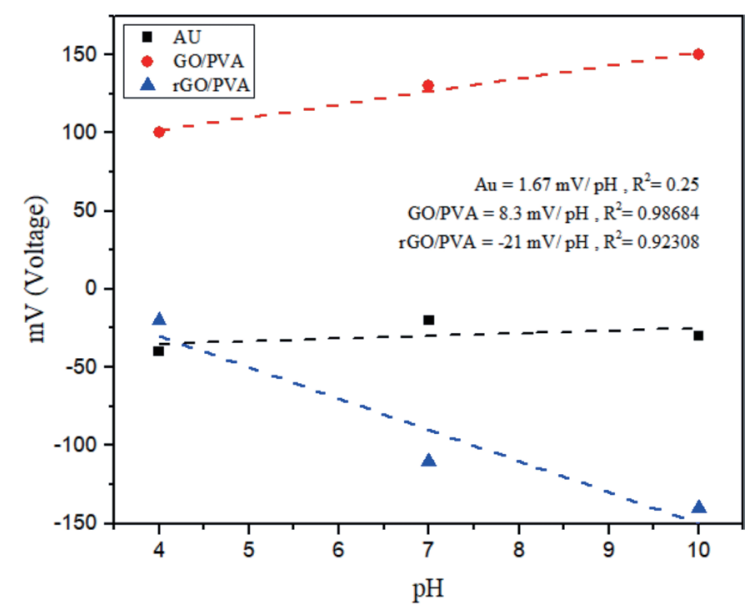

(b)

Fig. 7. (Color online) (a) OCP measurements of Au, GO/PVA, and rGO/PVA at pHs 4, 7, and 10. (b) Linear regression for measured OCP on $\mathrm{Au}, \mathrm{GO} / \mathrm{PVA}$, and rGO PVA.

When the sensor is immersed in the buffer solution, the electrodes interact with the ionic species present. The movement of ions involves the diffusion of ionic species into the electrode material, which produces a change in current. According to Ohm's law, a potential difference must be present when current flow exists. Consequently this gave rise to the data in Fig. 7(a).

In Fig. 7(a), the OCP results were obtained from sensors with WEs of Au, GO/PVA, and $\mathrm{rGO} / \mathrm{PVA}$. At a glance, the measured potential from GO/PVA is positive throughout the $\mathrm{pH}$ ranges, additionally being directly proportional to $\mathrm{pH}$. Both the $\mathrm{Au}$ and $\mathrm{rGO} / \mathrm{PVA}$ electrodes showed otherwise, with the measured potential being negative in all $\mathrm{pH}$ ranges. One stark difference between $\mathrm{Au}$ and $\mathrm{rGO} / \mathrm{PVA}$ is that the former experienced insignificant changes across the studied $\mathrm{pH}$ range. This signified that $\mathrm{Au}$ is less susceptible to $\mathrm{pH}$ changes. In the case of the rGO/PVA electrode, the measured potential becomes more negative as $\mathrm{pH}$ increases. However, it can be noted that the rise time for $\mathrm{pH}$ sensing is considerably longer than that of $\mathrm{Au}$ and GO/PVA.

Figure 7(b) shows the measured OCP for all sensors. Here, a linear model was adopted and the relationship between OCP and $\mathrm{pH}$ can be approximated as the gradient of the OCP profile. $\mathrm{Au}$ exhibited the least change in OCP against $\mathrm{pH}$ (about $1.67 \mathrm{mV} / \mathrm{pH}$ ). This is followed by $\mathrm{GO} / \mathrm{PVA}$ with $\sim 8.3 \mathrm{mV} / \mathrm{pH}$. Although $\mathrm{rGO} / \mathrm{PVA}$ shows the greatest change, i.e., $-21 \mathrm{mV} / \mathrm{pH}$, the relationship is inverse to that of $\mathrm{Au}$ and GO/PVA. Also, more time was needed to stabilise the OCP as shown in Fig. 7(a). In short, among the three sensors, GO/PVA electrodes is practical in terms of $\mathrm{pH}$ sensing. Table 3 shows the previous studies on using carbon-based materials. These interesting findings proved that weak $\mathrm{pH}$ sensitivity is not surprising when using carbonbased materials.

The sensing mechanism can be attributed to the charge transfer involving adsorbed $\mathrm{H}^{+}$ on the surface of the electrodes. This would become more efficient if the surface of the electrode contained adducts that permit such adsorption. Since the Au surface was composed 
Table 3

Comparison of various works on $\mathrm{pH}$ sensors.

\begin{tabular}{lcc}
\hline Electrode material & $\mathrm{pH}$ sensitivity $(\mathrm{mV} / \mathrm{pH})$ & Reference \\
\hline Suspended graphene & $\sim 20$ & Cheng et al. ${ }^{(20)}$ \\
\hline Suspended graphene & $\sim 18$ & Heller et al. ${ }^{(21)}$ \\
SWNT & -7.3 & Sohn et al. ${ }^{(18)}$ \\
\hline rGO FET & $\sim 29$ & Fu et al. ${ }^{(22)}$ \\
\hline Graphene FET & $\sim 6$ & \\
$\mathrm{Au}$ & 1.67 & This work \\
$\mathrm{GO} /$ PVA & $\sim 8.3$ & \\
rGO/ PVA & -21 &
\end{tabular}

of only pristine $\mathrm{Au}$, which lacks functional groups, the sensitivity was expected to be small. Meanwhile, a comparison of GO and rGO revealed that the former would have more oxygencontaining functional groups than the latter. ${ }^{(23)}$ The common functional groups that both materials possessed are the carboxylic $(-\mathrm{COOH})$ and hydroxyl $(-\mathrm{OH})$ groups decorating the edges. These groups are susceptible to protonation/deprotonation under various $\mathrm{pH}$ conditions. ${ }^{(24)}$

In an acidic medium, the negatively charged functional groups on GO/PVA are preferably protonated, resulting in free carriers that will diffuse towards the Au surface underneath, subsequently completing the circuit. As $\mathrm{pH}$ increases, protonation towards the functional groups becomes less efficient since the groups will undergo deprotonation, the effects of which should dominate under typical conditions. However, instead of expecting a decrease in OCP, the measured OCP was found to be increasing. It is considered that PVA plays an essential role here because it can form a polymerised matrix that would hold the ensemble of GO together. ${ }^{(25)}$ PVA in general also possesses functional groups similarly to GO. However, given their interaction with GO in terms of forming a polymerised network, the functional groups of PVA would be in direct contact with the buffer solution, while being a permeable membrane. Under typical conditions, deprotonation preferably occurs on PVA, hence, allowing GO to detect $\mathrm{H}^{+}$. This eventually leads to electrical polarisation on the GO/PVA interface, which inadvertently increases OCP.

\section{Conclusions}

A three-electrode configured $\mathrm{pH}$ sensor has been successfully fabricated, with WE being either $\mathrm{Au}, \mathrm{GO} / \mathrm{PVA}$, or rGO/PVA. The difference between GO and $\mathrm{rGO}$ can be observed from the Raman spectra for different values of $I_{D} / I_{G}$. When the electrode performances were evaluated by $\mathrm{CV}$, all of them exhibited a reversible redox reaction, indicating good chemical reactivity. Several differences between GO/PVA and rGO/PVA WEs have been noted, i.e., peak current magnitude and redox potential. The anomalous low peak current for rGO/PVA is indicative of other significant mechanisms in play despite $\mathrm{rGO}$ possessing a higher intrinsic electrical conductivity than GO. It was concluded that the oxygen-containing functional groups within GO, rGO, and PVA played an essential role in $\mathrm{pH}$ sensing. The chemical nature of these functional groups was affected by the $\mathrm{pH}$ of the medium. In this study, it was found that 
the GO/PVA electrode showed better sensing capability owing to an abundance of functional groups. Interestingly, the increase in OCP for GO/PVA along with $\mathrm{pH}$ suggests that PVA interacts with the buffer solution while allowing GO to detect the change in $\mathrm{H}^{+}$ions within the solution. Nevertheless, the $\mathrm{pH}$ sensing capabilities of all three types of WE have been compared and their mechanism of detection could be incorporated into other applications, such as heavy metal ion sensing, in the future.

\section{Acknowledgements}

The author would like to thank Universiti Sains Malaysia Research University for all the research facilities provided for this project. This work was supported by Top down Research University (USM) grant number 1001/PCEDEC/870050 and Bridging grant 304/ PCEDEC/6316088.

\section{References}

1 Y. Wang, H. Xu, J. Zhang, and G. Li: Sensors 8 (2008) 2043. https://doi.org/10.3390/s8042043

2 K. E. Zinoviev, A. B. González-Guerrero, C. Domínguez, and L. M. Lechuga: J. Lightwave Technol. 29 (2011) 1926. https://doi.org/10.1109/jlt.2011.2150734

3 A. C. Sun and D. A. Hall: Electroanalysis 31 (2019) 2. https://doi.org/10.1002/elan.201800474

4 R. J. Forster and T. E. Keyes: Handbook of Electrochemistry: Ultramicroelectrodes (Elsevier, Amsterdam, 2007) 1st ed., Chap. 6.

5 D. Sharma, J. Lee, J. Seo, and H. Shin: Sensors 17 (2017) 2128. https://doi.org/10.3390/s17092128

6 F. He, Y. Xiong, J. Liu, F. Tong, and D. Yan: Biosens. Bioelectron. 77 (2016) 799. https://doi.org/10.1016/ j.bios.2015.10.054

7 S. Partel, S. Kasemann, P. Choleva, C. Dincer, J. Kieninger, and G. A. Urban: Sens. Actuators, B 205 (2014) 193. https://doi.org/10.1016/j.snb.2014.08.065

8 C. Ma, N. M. Contento, L. R. Gibson, and P. W. Bohn: ACS Nano 7 (2013) 5483. https://doi.org/10.1021/ nn401542x

9 Z. Ramshani, A. S. Reddy, B. B. Narakathu, J. T. Wabeke, S. O. Obare, and M. Z. Atashbar: Sens. Actuators, B 217 (2015) 72. https://doi.org/10.1016/j.snb.2014.12.026

10 F. Perdigones, J. M. Moreno, A. Luque, C. Aracil, and J. Quero: Biomedical Engineering, Trends in Electronics: Communications and Software (InTech, Rijeka, 2011) p. 45.

11 B. K. Wang, Y. A. Chen, A. Shorey, and G. Piech: Proc. 2012 7th International Microsystems, Packaging, Assembly and Circuits Technology Conf. (IEEE, 2012) 247-250.

12 E. W. Nery and L. T. Kubota: Anal. Bioanal.Chem. 405 (2013) 7573. https://doi.org/10.1007/s00216-013-6911-4

13 I. Hamzah, A. A. Manaf, and O. Sidek: J. Adhes. Sci. Technol. 27 (2013) 1122. https://doi.org/10.1080/0169424 3.2012.735194

14 N. Vasilakis, K. Papadimitriou, D. Evans, H. Morgan, and T. Prodromakis: Proc. 2016 IEEE Healthcare Innovation Point-ff-Care Technologies Conf. (IEEE, 2016) 126-129.

15 H. Cui, X. Xiong, B. Gao, Z. Chen, Y. Luo, F. He, S. Deng and, L. Chen: Electroanalysis 28 (2016) 2000. https://doi.org/10.1002/elan.201501153

16 S. K. Lee, B. H. Kim, and H. J. Yoo: J. Semicond. Tech. Sci. 9 (2009) 174. https://doi.org/10.5573/ jsts.2009.9.3.174

17 M. Mellon, J. Mangadlao, R. Advincula, and E. Pentzer: RSC Adv. 8 (2018) 18388. https://doi.org/10.1039/ c8ra03300e

18 I. Y. Sohn, D. J. Kim, J. H. Jung, O. J. Yoon, T. N. Thanh, T. T. Quang, and N. E. Lee: Biosens. Bioelectron. 45 (2013) 70. https://doi.org/10.1016/j.bios.2013.01.051

19 P. Sahoo, B. Panigrahy, S. Sahoo, A. K. Satpati, D. Li, and D. Bahadur: Biosens. Bioelectron. 43 (2013) 293. https://doi.org/10.1016/j.bios.2012.12.031

20 Z. Cheng, Q. Li, Z. Li, Q. Zhou, and Y. Fang: Nano Lett. 10 (2010) 1864. https://doi.org/10.1021/nl100633g

21 I. Heller, S. Chatoor, J. Männik, M. A. Zevenbergen, C. Dekker, and S. G. Lemay: J. Am. Chem. Soc. 132 (2010) 17149. https://doi.org/10.1021/ja104850n 
22 W. Fu, C. Nef, O. Knopfmacher, A. Tarasov, M. Weiss, M. Calame, and C. Schönenberger: Nano Lett. 11 (2011) 3597. https://doi.org/10.1021/nl201332c

23 S. Bandi, S. Ravuri, D. R. Peshwe, and A. K. Srivastav: J. Hazard. Mater. 366 (2019) 358. https://doi. org/10.1016/j.jhazmat.2018.12.005

24 G. Kime, K. G. Zhou, S. J. Hardman, R. R. Nair, K. S. Novoselov, D. V. Andreeva and D. J. Blinks: J. Phys. Chem. C 123 (2019) 10677. https://doi.org/10.1021/acs.jpcc.9b01060

25 L. H. Sinh, N. D. Luong, and J. Seppälä: Plast. Rubber Compos. 48 (2019) 466. https://doi.org/10.1080/1465801 1.2019.1664820 